\title{
المجتمع المدني ودوره في التعديل الدستوري
}

Civil society and its role in the constitutional amendment

\author{
يوسف أزروال.جامعة باتنة.الجزائر
}

Available online at https://www.maspolitiques.com/ojs/index.php/ajps/article/view/60

\begin{abstract}
The concept of civil society was distinguished by new meanings that were clearly manifested in political thought, through the development of the concept of the nation-state to the modern state, and the transformations that took place on the latter affected the structure of the international system, and the implications of globalization in its various forms, as well as the impact of the digital revolution and the development of information and communication technology. These implications had multiple ideological dimensions, mainly directed towards neoliberalism, which called for reducing the role of the state and limiting its interference in the economic field, and from it its impact extended to the political field, this opened the way for the third sector (civil society) to contribute to the process of political development and the transformation industry, This is done through playing a central role in the constitutional reform process, as well as to complete the process of democratic construction, deepening the strips of peaceful and sound democratic political practice, and creating an open political community that believes in an effective democratic value system.
\end{abstract}

Key words: peaceful, civil society, constitution, democracy, governance . 
ملخص:

تميز مفهوم المجتمع المدني بمدلولات جديدة تجلت بشكل واضح في الفكر السياسي، وذلك عبر تطور مفهوم الدولة القومية إلى الدولة الحديثة، وما انجر عن هذه الأخيرة من تحولات مست بنية النظام الدولي، وتداعيات العولمة بمختلف صورها، فضلا عن أثز الثورة الرقمية وتطور تكنولوجيا المعلومات والاتصال. وقد كان لتلك المدلولات أبعادا أيديولوجية متعددة، اتجهت أساسا نحو النيولييرالية الداعية إلى تقليص دور الدولة والحد من تدخلها في الدجال الاقتصادي، ومنه امتد أثزها إلى الحقل السياسي، هذا ما فتح المجال أمام القطاع الثالث(المجتمع المدني) للمساهمة في عملية التتمية السياسية وصناعة التحول، وذلك من خلال الاضطلاع بدور مركزي في عملية الإصلاح الدستوري، وكذا لاستكمال عملية البناء الديمقراطي، وتعميق شرائط الممارسة السياسية الديمقراطية السلمية والسليمة، وخلق مجتمع سياسي مفتوح بؤمن بشكل فعال بالمنظومة القيمية الديمقراطية . الكلمات المفتاحية:سلمية،مجتمع مدني،دستور ،ديمقراطية،جكم. 
جاءت هذه الورقة البحثية تبتغي تفكيك حدود وأنماط العلاقة بين السلطة والمجتمع المدني، أب اثتغاله

من أجل الحد من سلطة الدولة وكبح جماح التعسف السياسي من جهة، وكذا تعزيز ودعم المشاركة السياسية للأفراد ومختلف المؤسسات الاجتماعية في مجال التعديل والإصلاح الدستوري من جهة أخرى. ومن هذا المنطلق بلورت إثكالية الورقة كما يلي : -كيف ساهم المجتمع المدني باعتباره أحد البنى الأساسية للنظام السياسي في تحقيقه لمطالب المجتمع

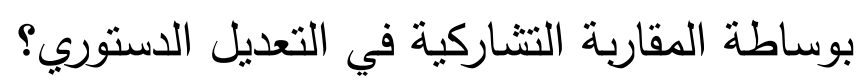
وتتدرج تحت هذه الإشكالية التساؤلات التالية: -ما هو مفهوم المجتمع المدني في ضوء الأطياف الفكرية المتعددة؟ -كيف ظهر المجتمع المدني في الجزائر؟ وما هي روافده؟ -ما المقصود بالتعديل الدستوري؟

-كيف ساهمت فواعل المجمع المدني في مناقنة مسار الاصطلاحات السياسية في الجزائر؟ وللإجابة على الأفكار المستشكلة أعلاه، ارتأيت اعتماد المحاور الآتية:

$$
\begin{aligned}
& \text { أولا: المجتمع المدني:مقاربة ايتمولوجية(المفاهيم، المقومات. ( } \\
& \text { ثانيا:المجتمع المدني في الجزائر (ظروف التأسيس، وروافده.) }
\end{aligned}
$$

تالثا:المجتمع المدني ودوره في التعديل الدستوري: مقاربة تتاركية.

ومن أجل فحص علاقة الدولة بالمجتمع المدني في سياق الإصلاحات السياسية، استخدمت

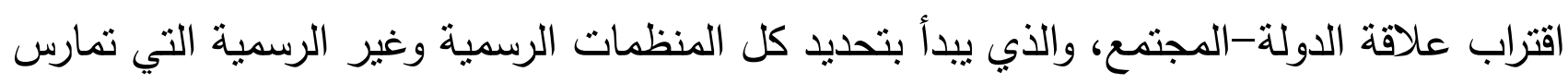

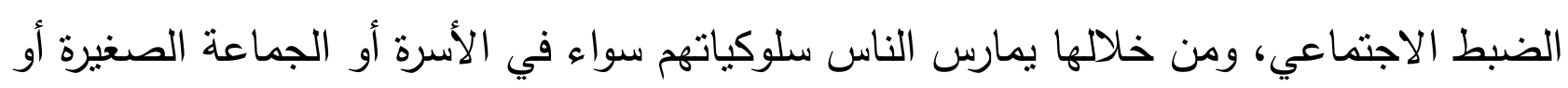

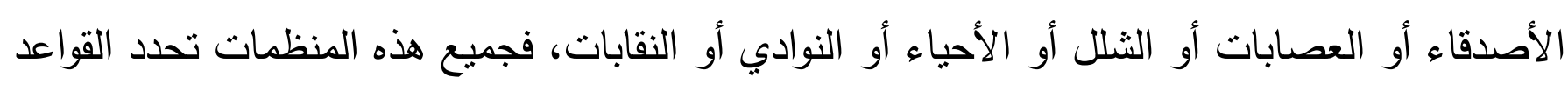

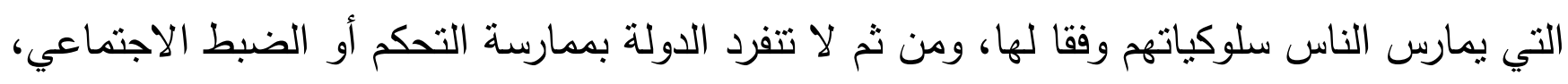
إنما تقوم هذه الجماعات أيضا بتحديد معايير للسلوك خارج القانون، أو المعايير التي وضعتها الدولة دون أن يعني هذا خروجا عن القانون.

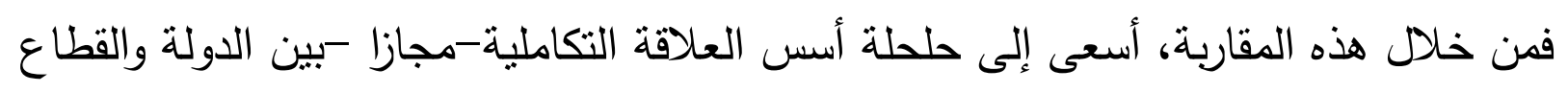
الثالث، أين فتحت المجال لروافده قصد المشاركة والإدلاء بدلوها من معين الإصلاحات السياسية الثها 
والتشريعية، وكذا طريقة تفاعل وتجاوب منظمات المجتمع المدني مع مخرجات النظام السياسي. أولا: المجتمع المدني:مقاربة ايتمولوجية(المفاهيم، المقومات. أ/ مفهوم المجتمع المدني: عرف المجتمع المدني كغيره من المفاهيم في الفكر السياسي تغيرات عدة،

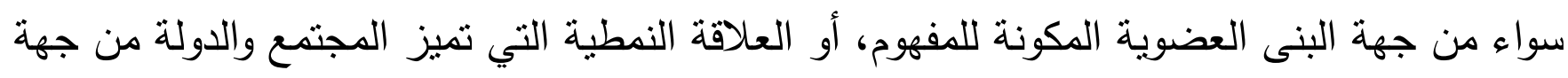
أخرى. وتعبر هذه العلاقة في فحواها عن وجود حالة من الاستقطاب بين المجتمع والسياسة، حيث

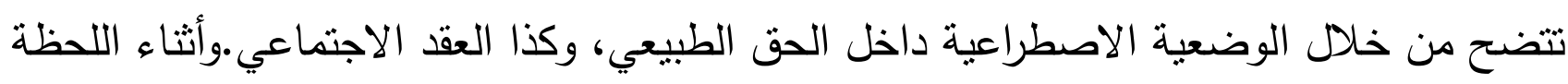

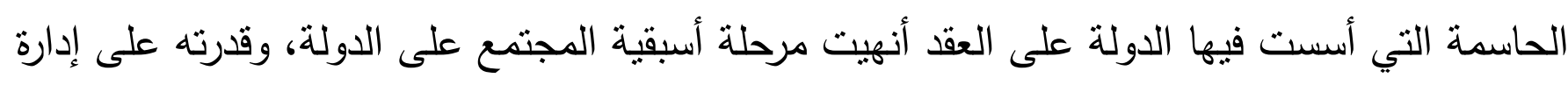

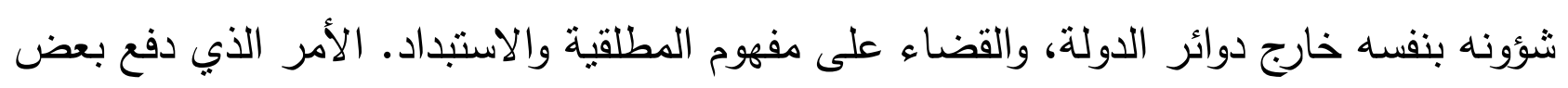
المفكرين إلى اعتباره مفهوم تشوبه الضبابية والغموض، بل وصل الأمر إلى اعتباره مفهوم زئبقي.

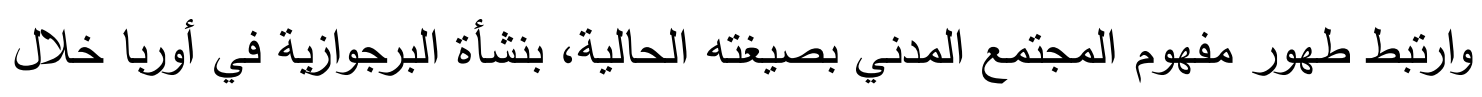
القرنين17و 18، وذللك عندما بدأت تكتسب الثروة والاستقلال فراحت تطالب بالحرية والتوسيع الحقوقي. ومع هذا فقد أعادت ظاهرة العولمة صياغته وإفرازه وإحياءه وفرضه كأداة للتعامل اليومي داخل البيئة الدولية والمجتمعية وبين الأفراد والمؤسسات، نتيجة للتحولات السياسية والاقتصادية، مما جعله يتأثز

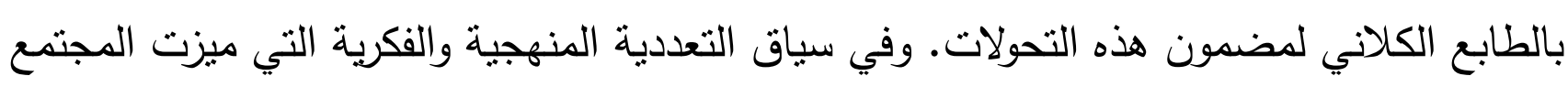

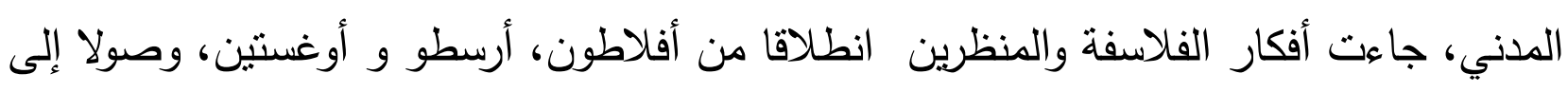

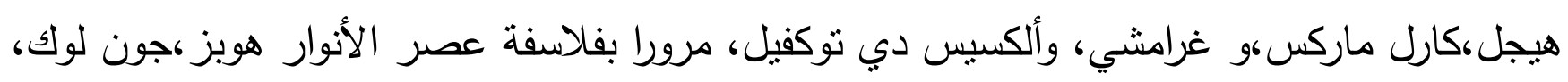
وجون جاك روسوو • فالمفكر الاتجليزي توماس هوبز يرى بأن المجتمع المدني هو المجتمع القائم على التعاقد، ولو اتخذ

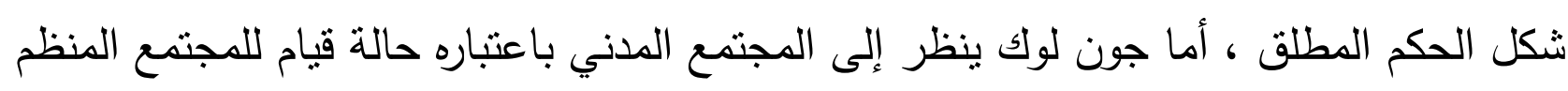

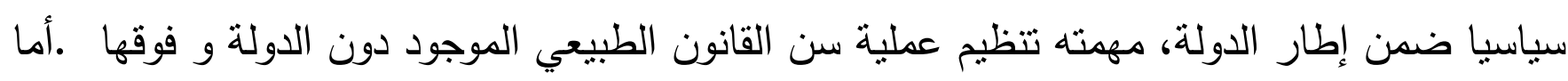
ج ج روسو يثير إلى تموقع المجتمع المدني بين الدولة وبقية عرى المجتمع، حيث يعتبره مجتمع صاحب سيادة، باستطاعته صياغة إرادة عامة يتماهى فيها الحكام والمحكومين.

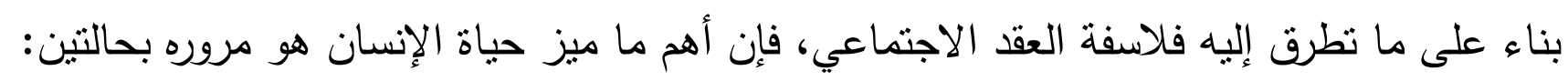
الأولى؛ حالة كان عليها قبل أن يدخل في المجتمع، فكان يعيش في الطبيعة بموجب قوانين هذه الأخيرة

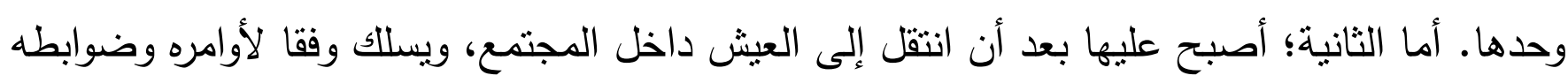

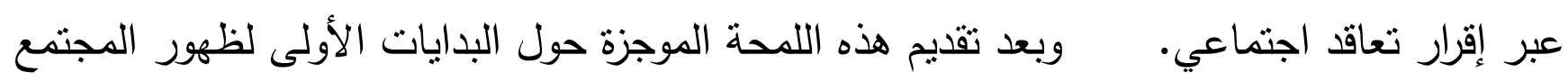




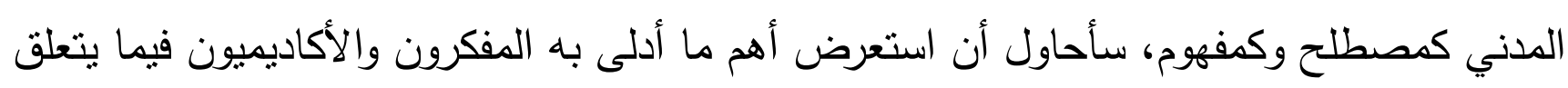
بالبنية المفهومانية لموضوع الدراسة، إلا أنه يجب التتويه إلى أن التعددية الدفاهيمية مرنبطة بالتوجهات

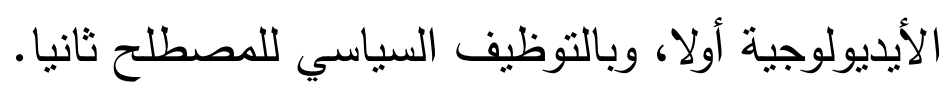

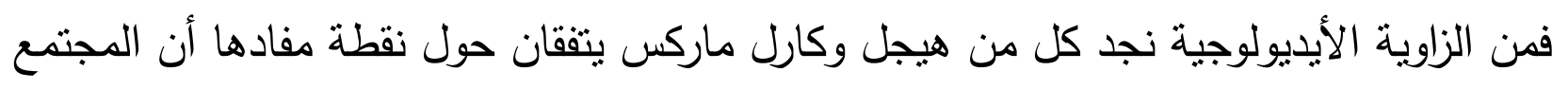

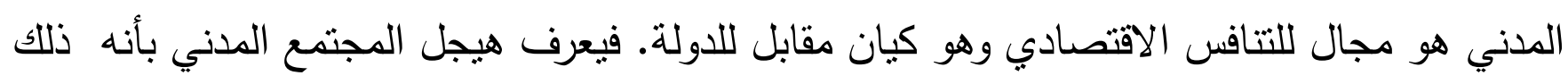

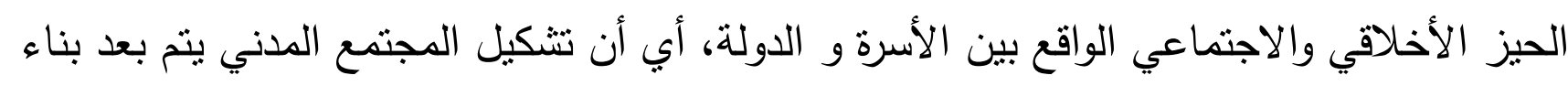

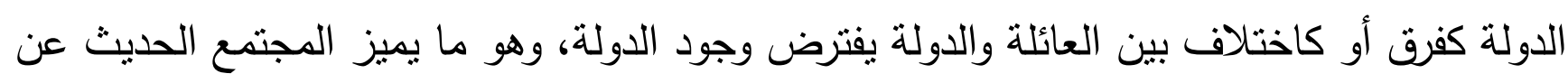

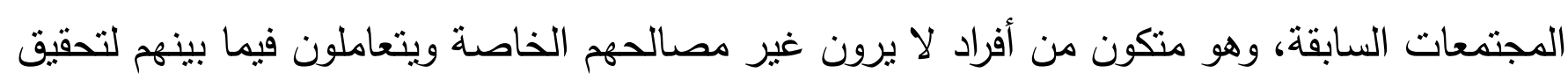

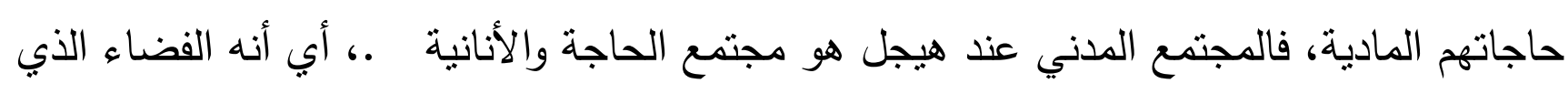

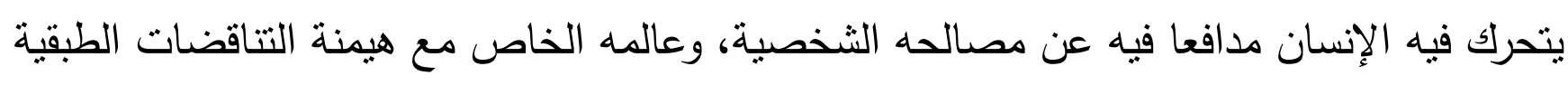

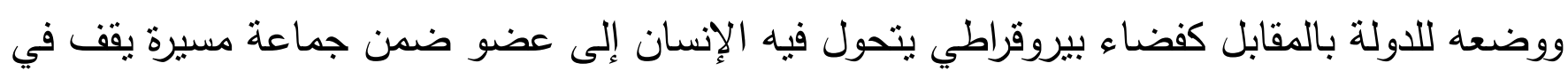
حقيقة الأمر غريبا بينها. أما كارل ماركس بنظر إلى المجتمع المدني بأنه الأساس الواقعي والمادي للاولة، وقد شخصه في

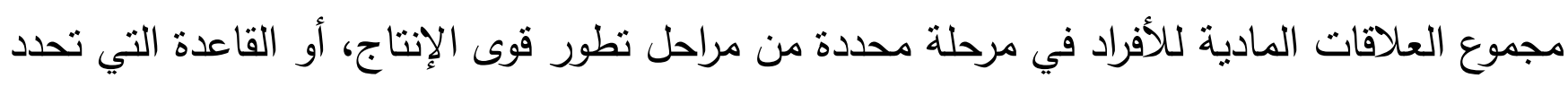

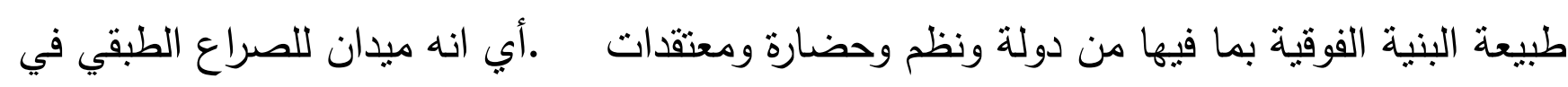

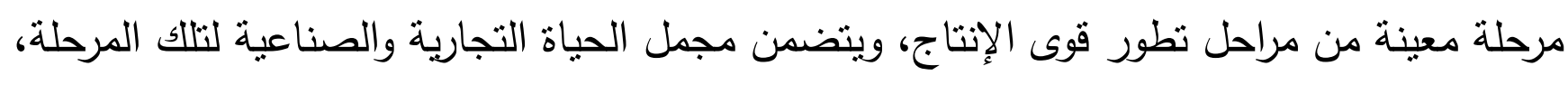

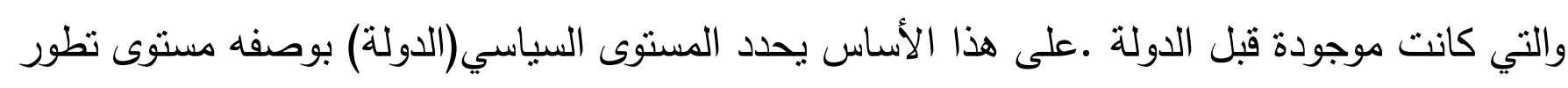

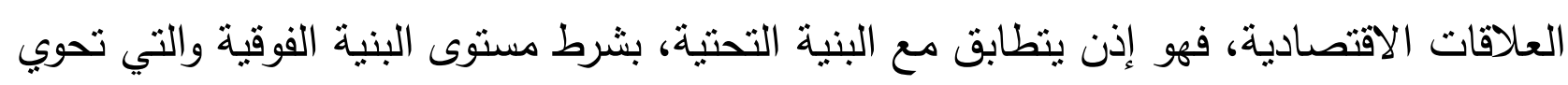
الايدولوجيا والمؤسسات السياسية.

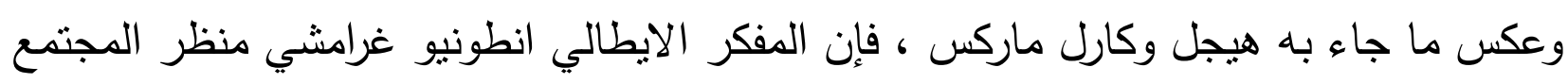
الددني، يعتبر المجتمع الدني مجالا للتنافس الإيديولوجي فهو فضاء تكون الأيديولوجيات المختلفة

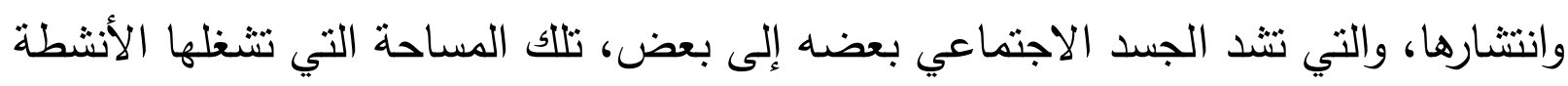

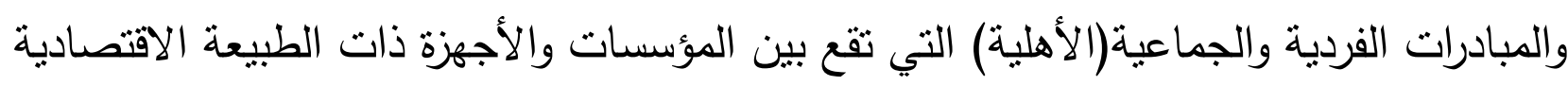

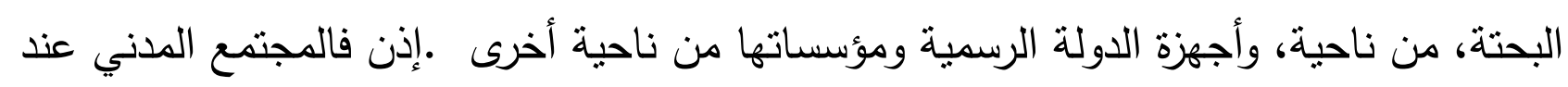

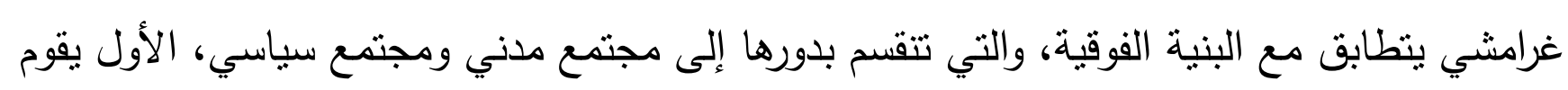
بالهيمنة عن طريق الثقافة و الأيديولوجيا، والثاني المنمتل في الدولة يقوم بوظيفة السيطرة والإكراه. 
وفي تعريف آخر للمجتمع الددني هو نسيج متثابك من العلاقات التي تقوم بين أفراده من

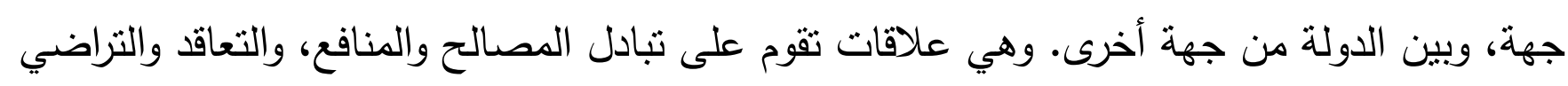

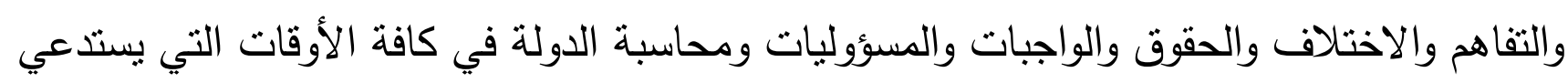

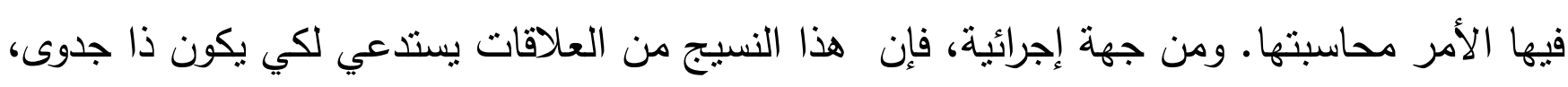

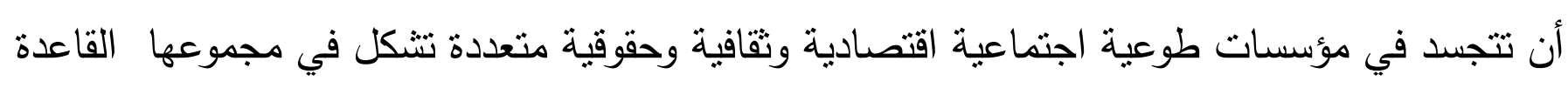

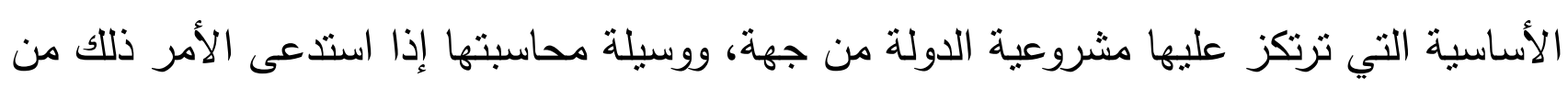

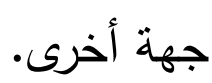

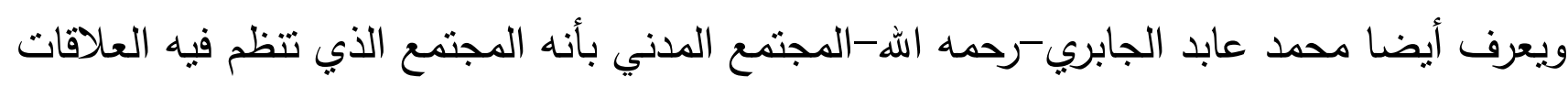

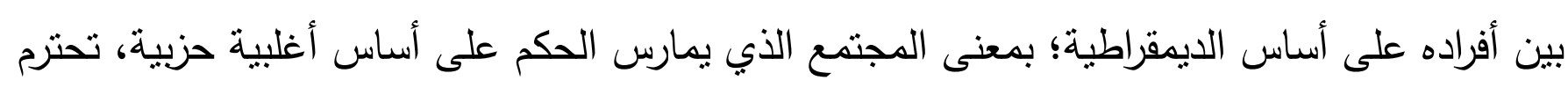

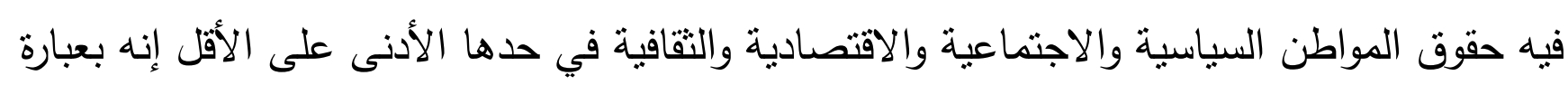

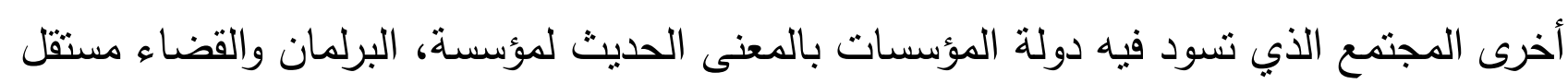
والأحزاب والنقابات والجمعيات.

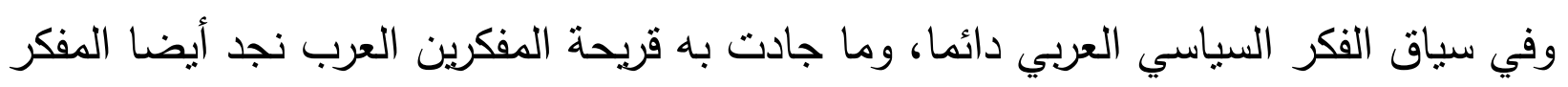

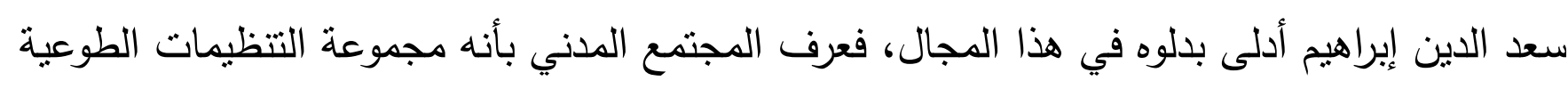

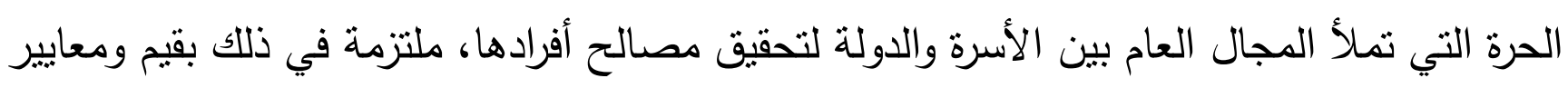

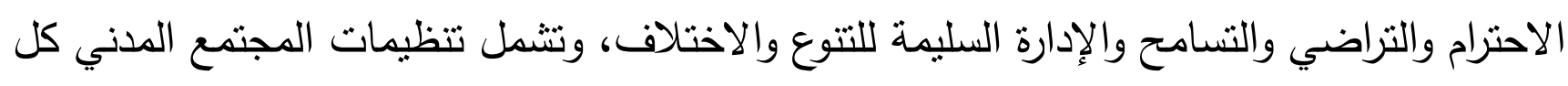

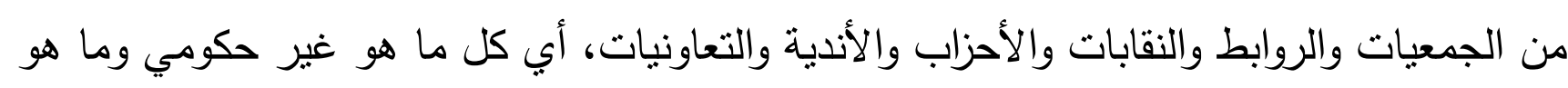
غير عائلي أو إرثي.

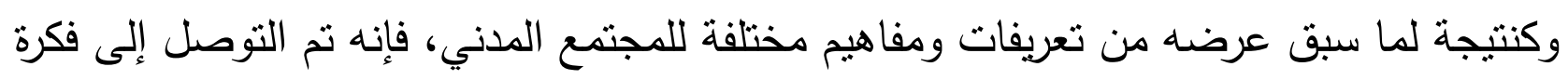

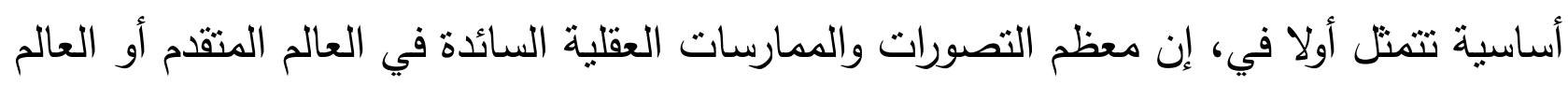

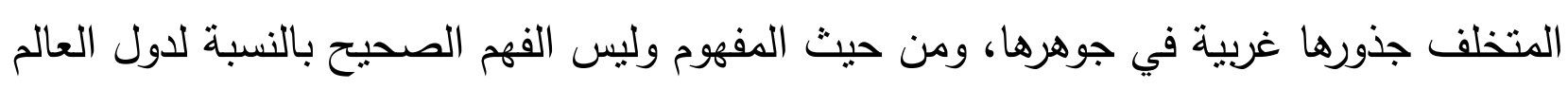

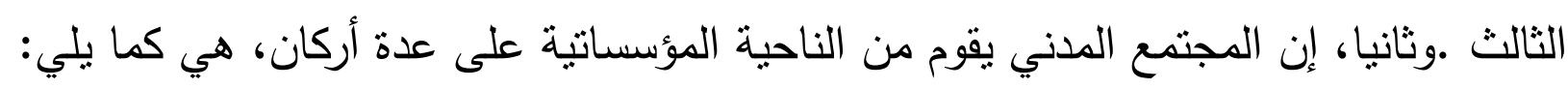
-1القدرة على التكيف: أب قدرة المؤسسة على التكيف مع التطورات التي تطرأ على مسنوى البيئة، فكلما تتمتع المؤسسة بالقدرة على التكيف كلما زادت فعاليتها، وللتكيف مستويات لفئ عدة:

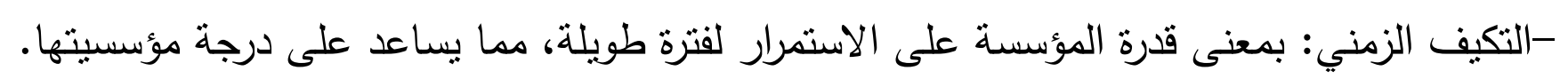
- التكيف الجيلي: يقصد به قدرة المؤسسة على الاستمرار مهما تعاقبت أجيال القيادات 
والزعامات عليها، أي لا يوجد صراع جيلي،كالصراع القائم بين جيلي الثورة الاستقلال على مستوى

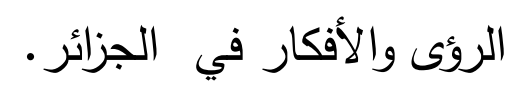

-التكيف الوظيفي: أي قدرة المؤسسة على إجراء تعديلات في أنشطتها ووظائفها. 2 -الاستقلالية:بقصد بها ألا تكون المؤسسة خاضعة لغيرها من المؤسسات أو الجمعيات أو الأفراد أو تابعة لها، حيث يسهل السيطرة عليها وتوجه نشاطها، وتحدد درجة استقلالية المجتمع المدني من خلال مؤشرين هما :

- نشأة مؤسسات المجتمع المدني وحدود تدخل الدولة في العملية، فالأصل هو أن تتمتع المؤسسات بهامش من الاستقلالية عن الدولة. -الاستقلال المالي لمؤسسات المجتمع المدني، وبيرز ذلك من خلال تحديد مصادر تمويل المؤسسات، فهل مصدر التمويل ذاتي داخلي أو خارجي؟ -3 - التعقد: يراد به تعدد المستويات الرأسية والأفقية داخل المؤسسة، أي تعدد هيئاتها التنظيمية من ناحية، ووجود مستويات ترانبية داخلها وانتشارها الجغرافي على أوسع نطاق ممكن داخل المجتمع.

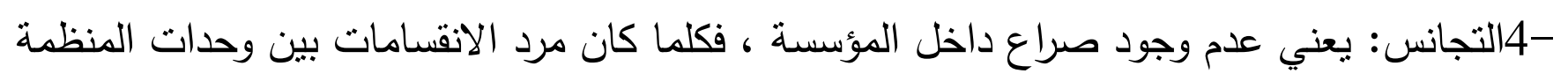
إلى أسباب تتعلق بنشاط المؤسسة، وكانت الطريقة لحل النزاع سلمية كان هذا دليلا على تطور المؤسسة.

وللمجتمع المدني بالغ الأهمية داخل الأنظمة السياسية، إذ تتضح أهميته على مستويين:

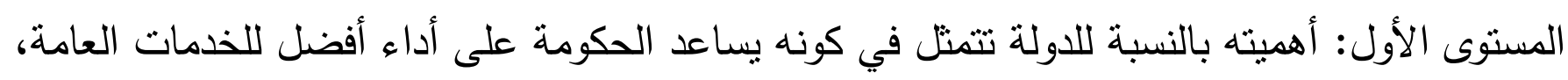
وكذا التأثير في رسم السياسات العامة، ودعم قيم المشاركة وتحقيق التتمية التشاركية. المستوى الثاني: أهميته بالنسبة للفرد(المواطن) لكونه يعمل على تربية المواطن على ثقافة الديمقراطية وإكساب أعضائها لقيم الحوار وقبول الآخر والاختلاف، وكذا مساءلة القيادات والمشاركة في الانتخابات والتعبير الحر عن الرأي، والمساهمة في صنع القرار وتعديل التشريعات داخل الدولة. ونظرا لأهمية المجتمع المدني باعتباره فاعل من الفواعل الأساسية التي تساهم في نرشيد أنظمة الحكم، سنحاول عبر المحور الموالي أن نتطرق إلى المجتمع المدني في الجزائر ، ثم نختتم الورقة البحثية بمحور يتتاول دور المجتمع المدني في التعديل الدستوري ومساهمته في الإصلاحات السياسية

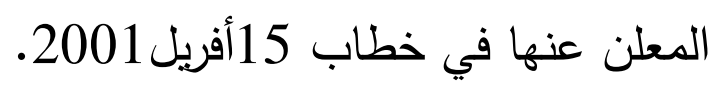


)

ثنانيا: المجتمع المدني في الجزائر(ظروف الثأسيس،روافده

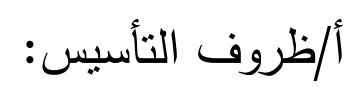

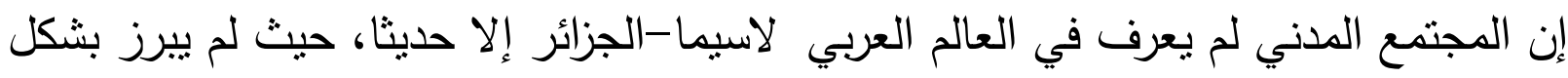

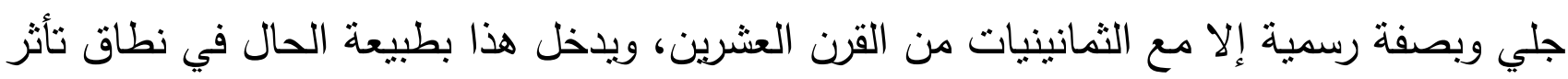

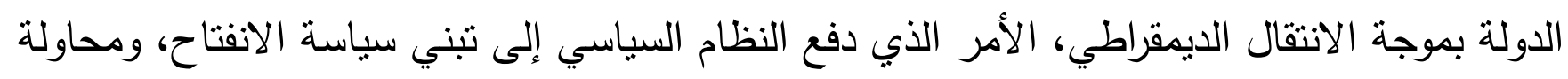
وضع اللبنات الأولى لعميلة النحول الايمقراطي.

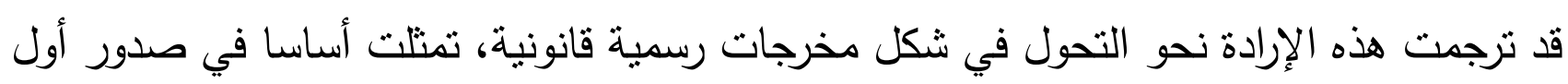

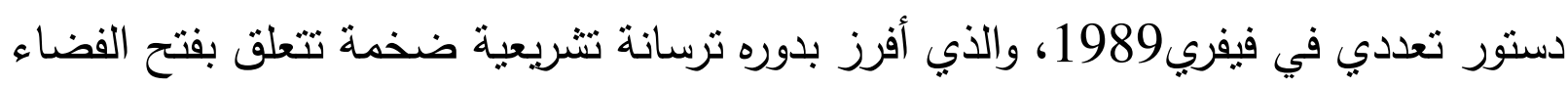

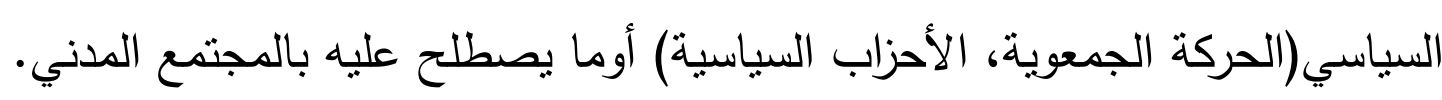

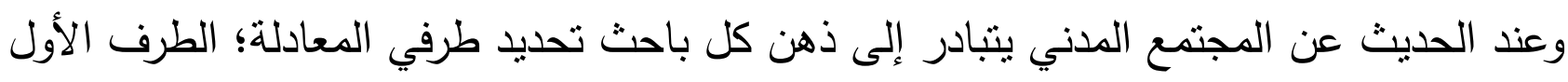

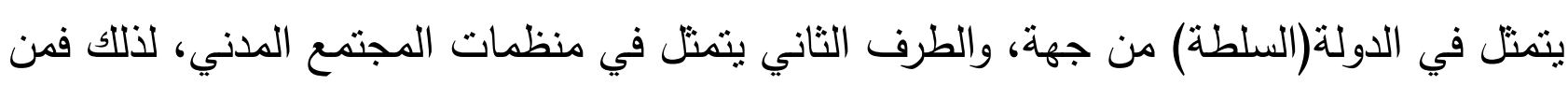
الضروري تنكيك العلاقة بين طرفي المعادلة.

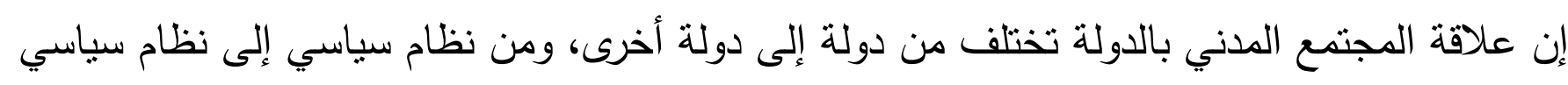

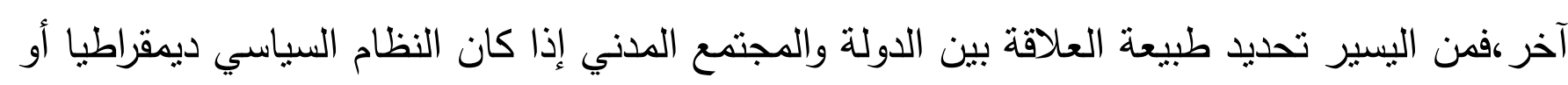

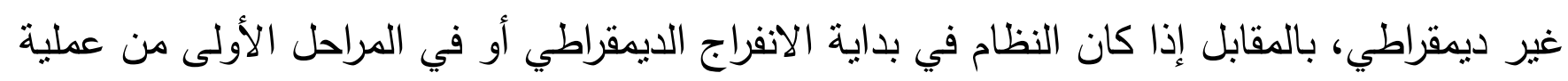

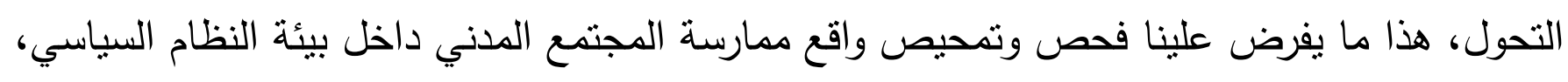

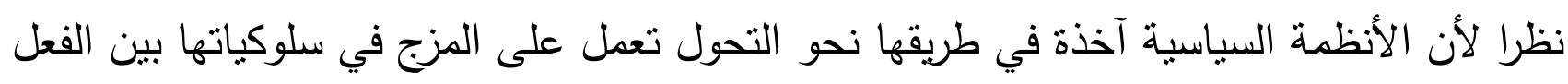

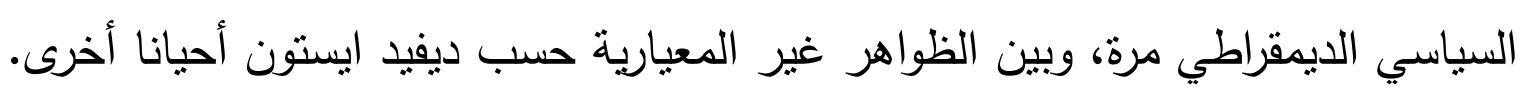

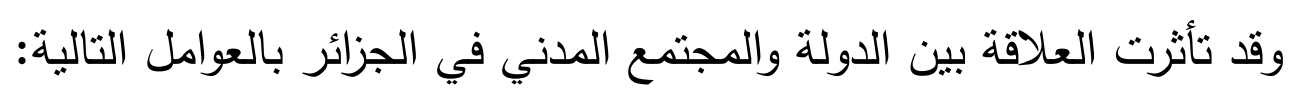

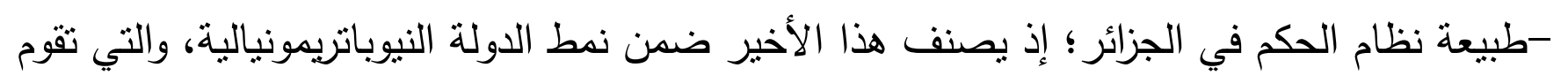

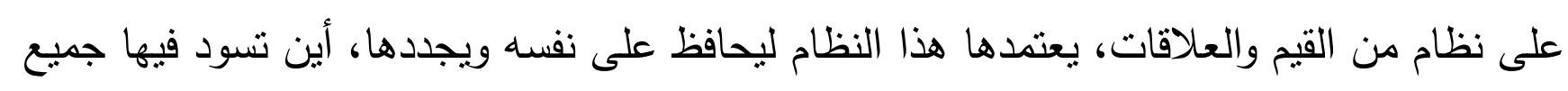

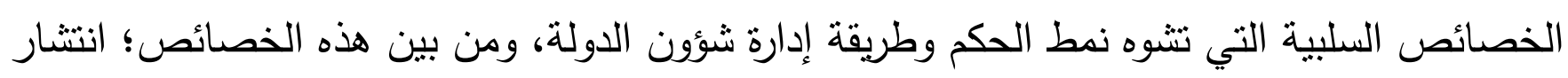
مظاهر الرشوة والعصبوية، والزيونية السياسية، والجهوية والمحاباة.

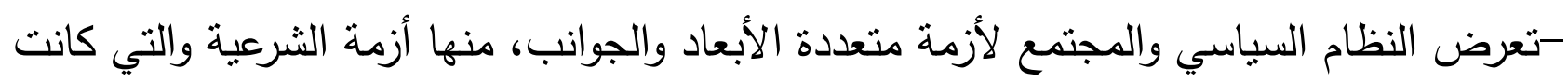

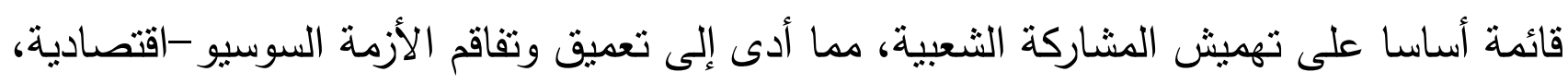

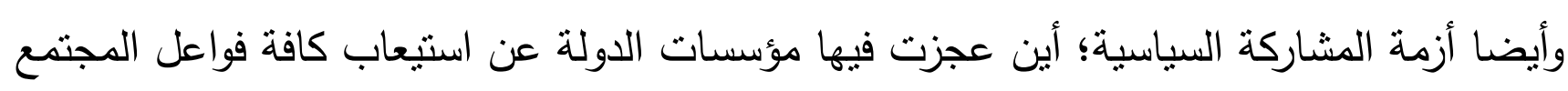


المدني، وسيطرة مفهوم التعبئة بدل المشاركة في العملية السياسية، وعلى مسنوى آخر نجد أن النظام

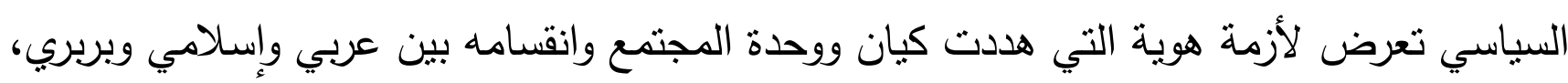
وأخيرا أزمة التوزيع؛ حيث تتمنل في توزيع الموارد والمنافع المادية وكذا توزيع عوائد التتمية. - ضعف الاستقلالية المالية لمنظمات المجتمع المدني، فأغلب الهبات والأغلفة المالية لمؤسسات القطاع الثالث ليست ذاتية نابعة من السياسة المالية لها، وإنما خارجية تابعة لدعم الدولة من جهة،

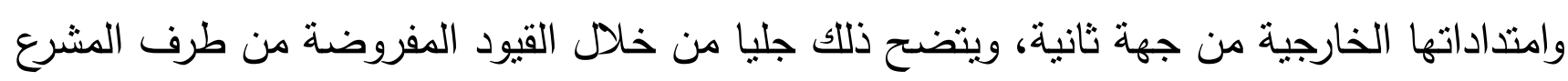

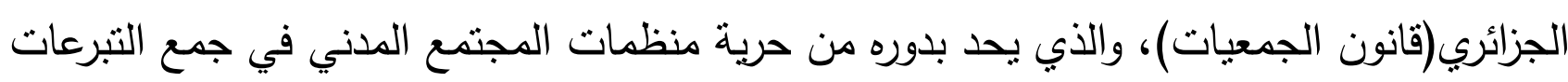
والمساعدات المالية المحلية والدولية، الأمر الذي يؤدي إلى الارتماء في أحضان السلطة، حيث تلثئ تصبح

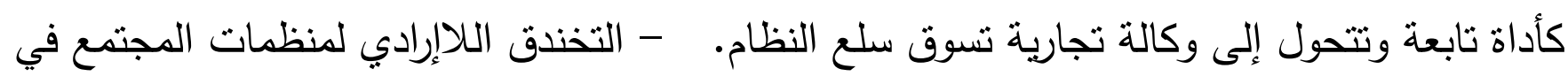
إطار السياسات المقررة من طرف دوائر النظام، نتيجة التضييق الممنهج الممارس من قبل السلطة، مما

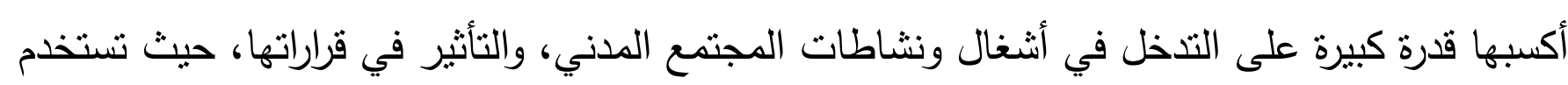

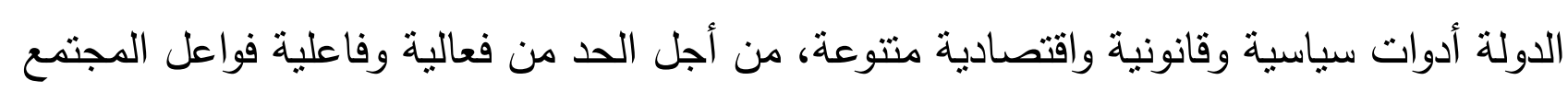

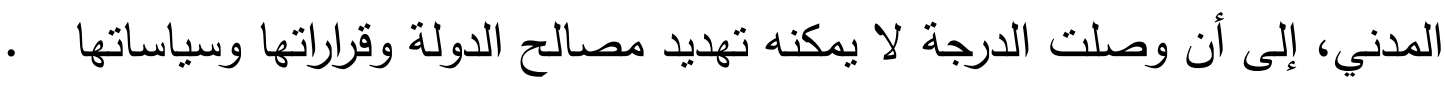
-ضعف المجتمع المدني مقابل هيمنة وتحكم الدولة، مما أضفى عليه ميزة التقابلية، أي مجتمع مدني

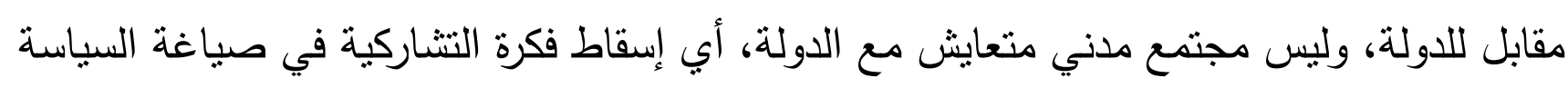

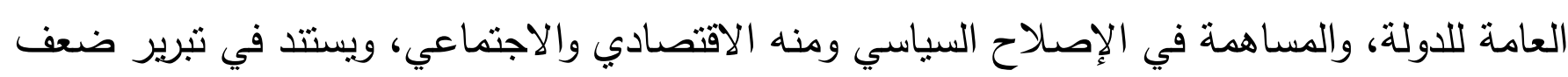

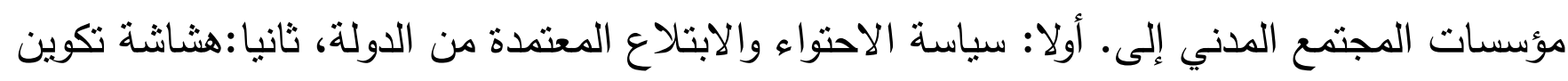
منظمات المجتمع المدني مما يجعله سهل الاختراق والانصهار بشكل كلاني في فللك أفكر السلطة. إن العوامل السابقة، تعبر بشكل أو بآخر عن ضبابية وتعقد العلاقة بين الدولة والمجتمع المدني، وهذا مرتبط أساسا-كما سبق وأن أشرت- إلى تزامن نشوء وتشكل المجتمع المدني في الجزائر بمرحلة الانتقال الديمقراطي، الأمر الذي ينجم عنه تحرير الفعل المدني لمختلف المؤسسات تارة، والتذخل توجيه

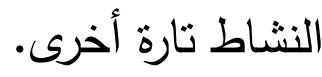

ب/روافد المجتمع المدني في الجزائر : بصدور دستور 23فيفري1989، والذي جاء على إثر تذهور

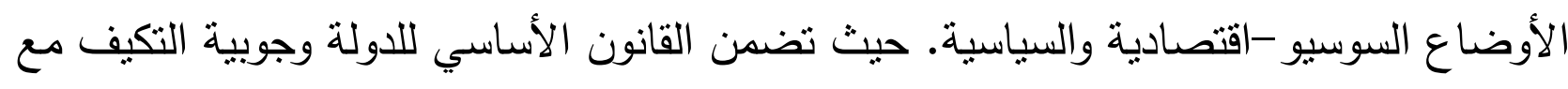
منطلبات التحول السياسي نحو منظومة القيم الديمقراطية، فبموجب هذا الدستور تم الانتقال من نظام

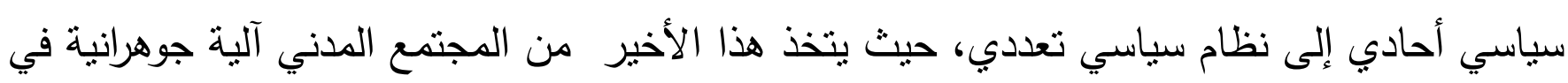


عملية التغيير الاقتصادي والاجتماعي والسياسي، وقبل نتاول علاقة المجتمع المدني بالدولة، ارتأيت أن

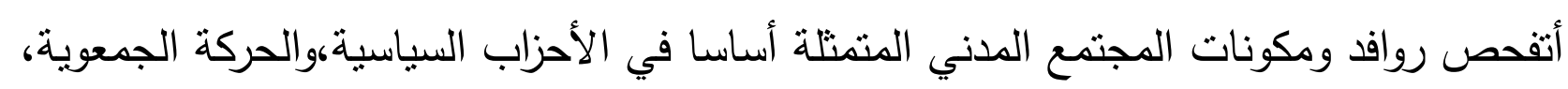

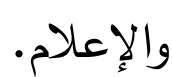

الرافد الأول: الأحزاب السياسية والانفتاح المقيد: يرجع ظهور الأحزاب السياسية داخل الفضاء السياسي

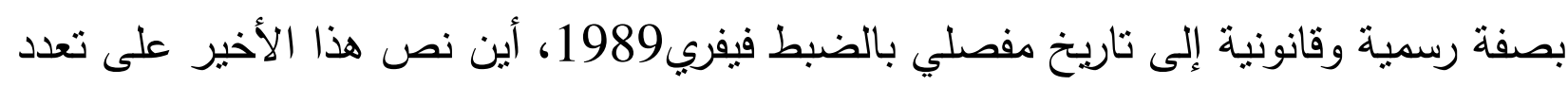

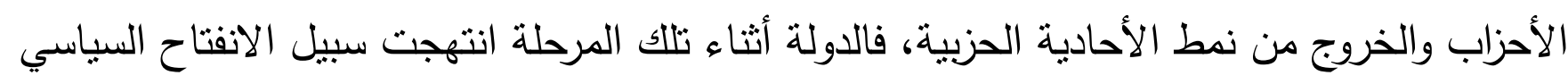

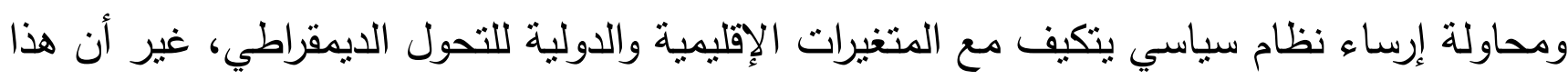

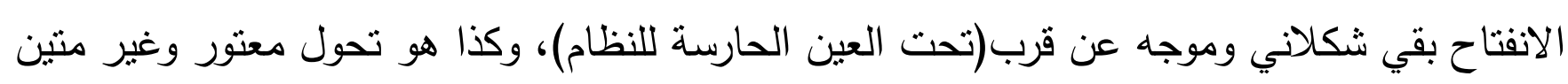

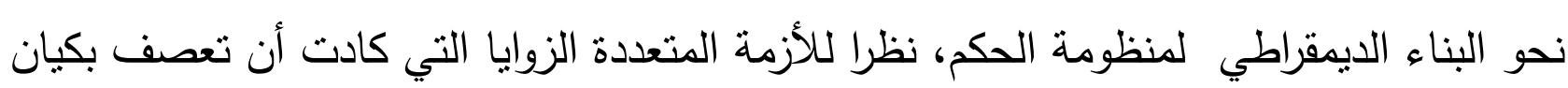

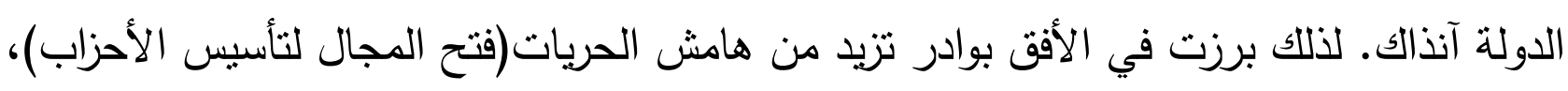

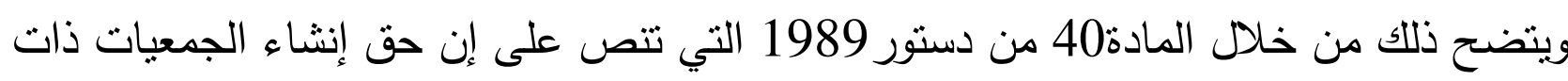

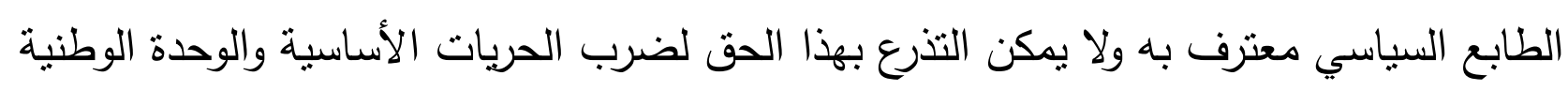

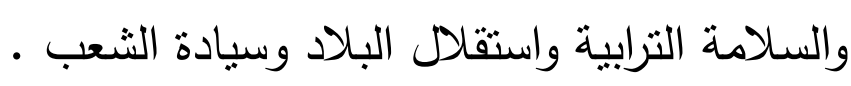

كما جاء دستورنوفمبر 1996 ليوضح أكثر ويحدد معالم وحدود الظاهرة الحزبية، حيث ميز بينها

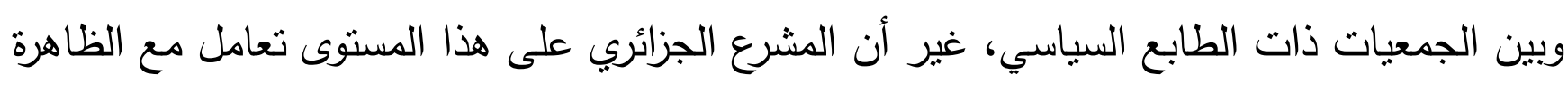

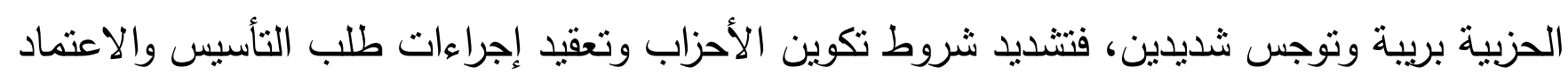

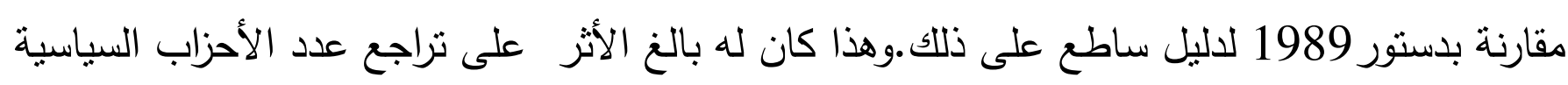

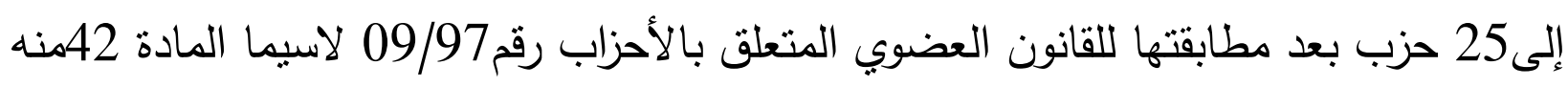

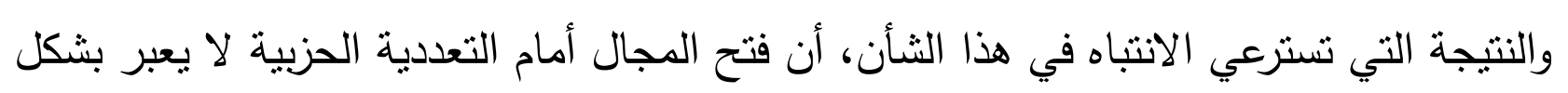

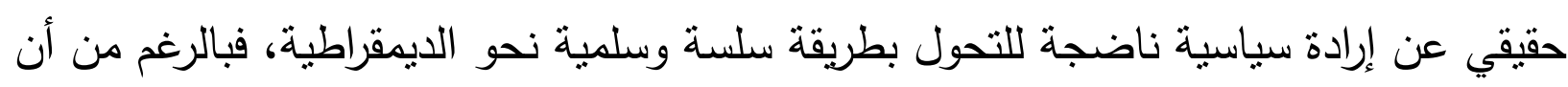

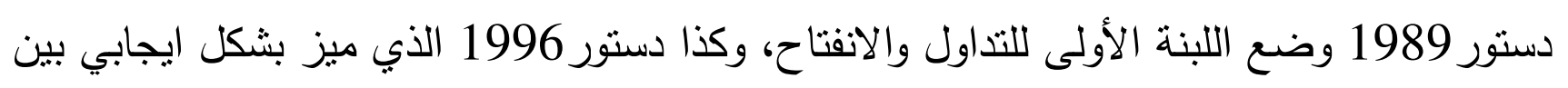
الحزب والجمعية ذات الطابع السياسي، فقد اتضح من الممارسة السياسية للنظام رفضه لآلية الانفتاح

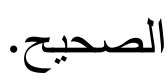

الرافد الثاني:الحركة الجمعوية: قد وسع القانون رقم31/90 من فرص تأسيس الجمعيات في مختلف

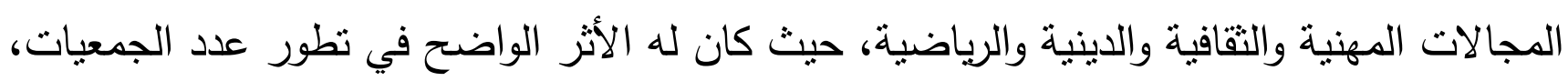

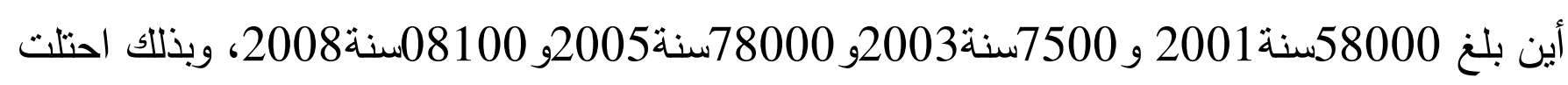


الجزائر الصدارة في الوطن العربي من حيث الترسانة العددية للحركة الجمعوية. الرافد الثالث: الإعلام(السلطة الرابعة): بمجرد الحديث عن قطاع الإعلام في الجزائر ، فإننا نقصد

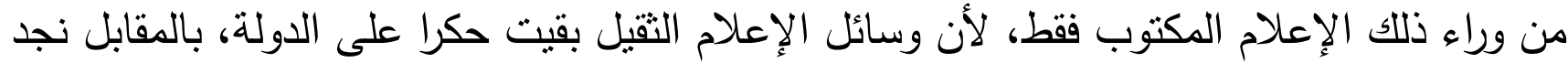
معظم دول العالم الديمقراطي فتحت الفضاء السمعي البصري للقطاع الخاص، أين أضحى الإعلام الخاص يؤدي دورا مهما في صياغة السياسة العامة للاولة، والمساهمة في تتشئة وتوعية المواطن الئن سياسيا، وذللك من خلال العمل على ردم وتقليص الفجوة بين الحاكم والمحكوم. وقد كان لقانون الإعلام الصادر سنة1990 بائن الأثر وبصفة ايجايية في تطور الإعلام على المستويين الإقليمي والمحلي، خاصة الإعلام في شقه المكتوب،حيث بلغ العدد الهائل للصحافة المكتوبة الصائ سقفا معتبرا، فنجد مثلا(400الف نسخة لجريدة الخبرو 500الف نسخة لجريدة الثروق و 150الف نسخة لجريدة البلاد و 150الف نسخة لجريدة الوطن و 200الف نسخة لجريدة يومية وهران. ومنه نستتنج، على الرغم من العدد المعتبر والتطور المتسارع والمضطرد لروافد المجتمع المدني،

فإنه لا يعكس المسؤولية المنوطة به على المستوى الأفقي وتغلغله في عمق القواعد النضالية، نظرا لأخذه صفة الرسمية الدولاتية أكثر منه سوسيو سياسية ملحة تزيد من دمقرطة النظام والحياة السياسية، والحد من تعسف السلطة في تعيم النظرة وفلسفة السلطة في إدارة شؤون الحكم، بمعنى أن المجتمع الدني في الجزائر انتقل من هيمنة الحزب الواحد في ظل الأحادية الحزبية إلى هيمنة السلطة في ظل العل

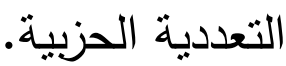

ثالثا:المجتمع المدني ودوره في التعديل الدستوري: مقاربة تتشاركية. إن التعديل الدستوري مسألة مقترنة في صلبها بالنسق العام للنظام السياسي، فهي تمس عمق فئقان النسق الدستوري القائم والسياسي أيضا بالدولة، وتسمح كذلك بتغيير ميزان التأثير الناظم لعلاقة طرفي

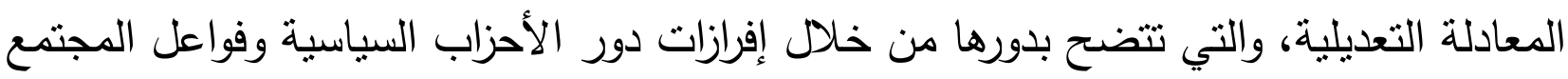
المدني، باعتباره رافعة ودعامة لباقي المؤسسات من جهة، ودوره في الحراك الاجتماعي والسياسي، والاقتصادي من جهة أخرى، وكذللك قدرته على الدساهمة بشكل فاعل في التطورات الدستورية في إطار إعادة صباغة علاقة الدولة بالمجتمع. وقبل الخوض في عرض دور المجتمع الددني في التعديل الدستوري، وجدت من الضرورة الإثنارة ولو بصفة موجزة لمفهوم التعديل الدستوري. تعريف التعديل الدستوري:إن التعديل الدستوري يعبر عن تغيير جزئي أو كلي شامل في أحكام الدستور ، 
سواء بإلغاء بعضها أو إضافة أحكام أخرى، والإلغاء الكلي لا يعبر بشكل أو بآخر عن تعديل الدستور،

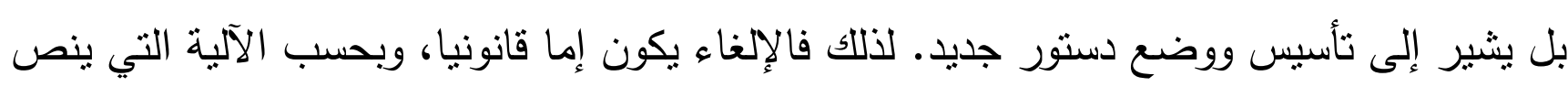
عليها الدستور، وإما يكون سياسيا عن طريق الظواهر غير المعيارية كالثورة والانقلاب والتمرد. فمن هذا الدنطلق، تتنهي القاعدة الدستورية، بأحد السبيلين: التعديل أو أو الإلغاء.

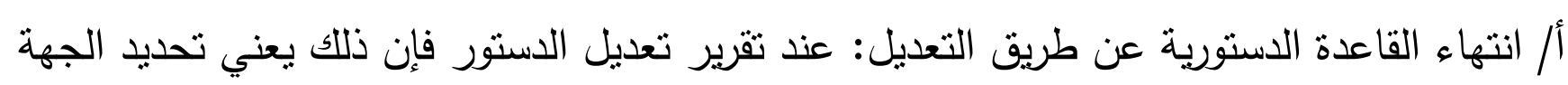

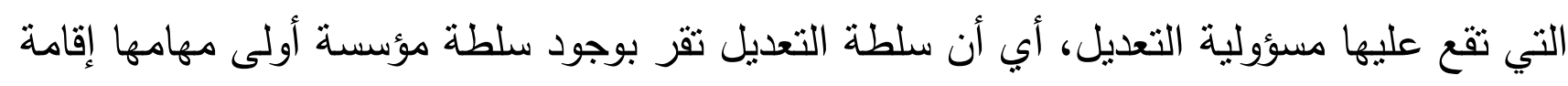

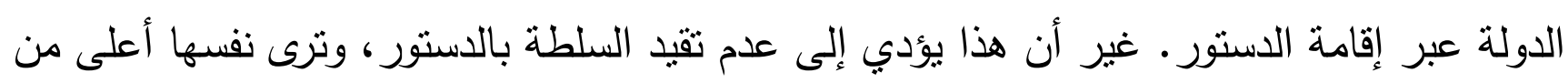

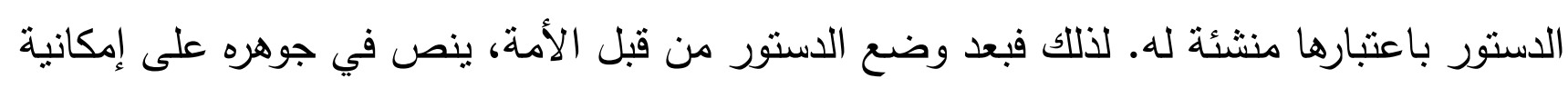

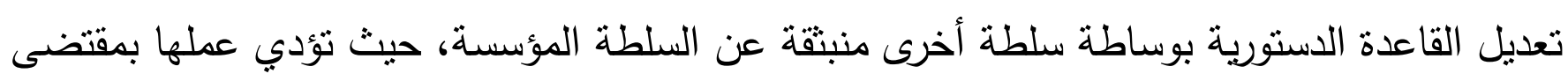

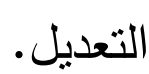

ب/ انتهاء القاعدة الدستورية بإلغاء الدستور : إن الإلغاء الكلي للاستور يكون أكثر انسجاما في حالة حدوثه، عندما لا تقوم الجهة نفسها التي وضعته بإلغائه كليا، ويلغى بطريقتين:

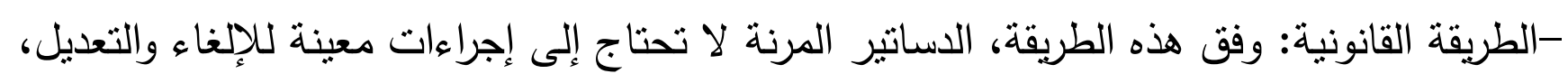
عكس الدساتير الجامدة التي تحتاج إلى إجراءات محددة وقابليتها للتعقد.

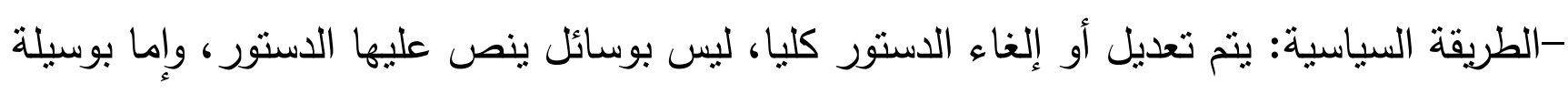

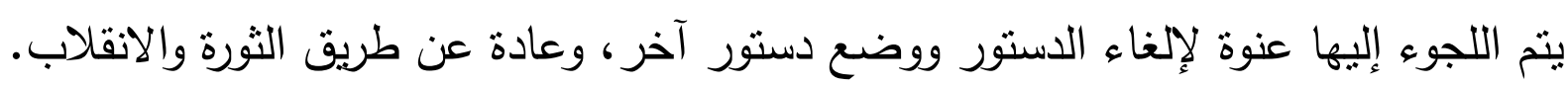

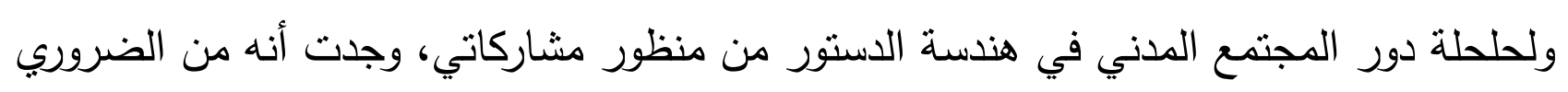

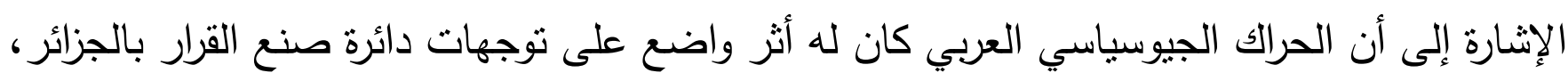

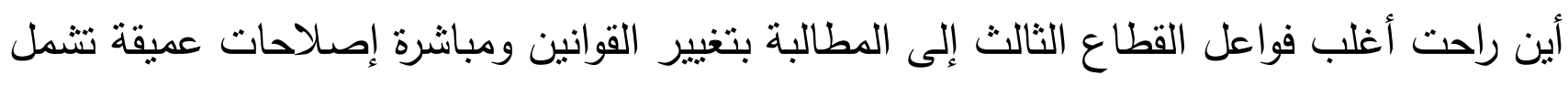

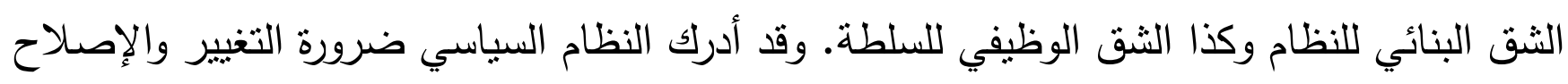

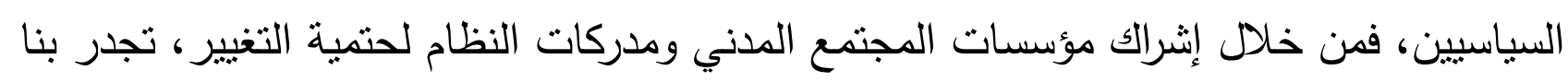

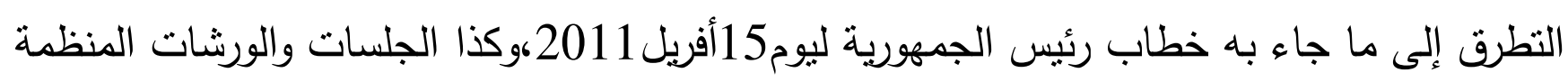
من طرف المجلس الوطني الاقتصادي والاجتماعي مع مختلف أطياف المجتمع المدني.

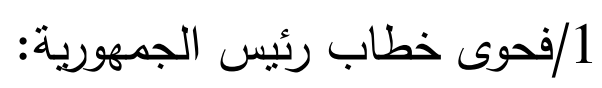

أ/إعادة صياغة العدة التثريعية: بعد تجربة التعددية والانفتاح السياسي والاقتصادي سنة 1989، آترت الإرادة السياسية بالجزائر إلى تصحيح مسار التحول دون التخلي و الدساس بالخيار التيار الديمقراطي. وقد 
جاء في هذا السياق خطاب رئيس الجمهورية يحرص فيه على توسيع فضاء المشاركة السياسية أمام

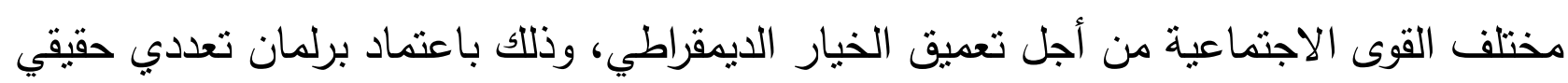

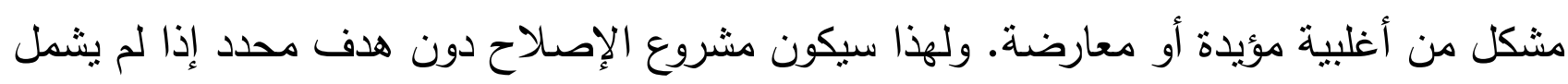

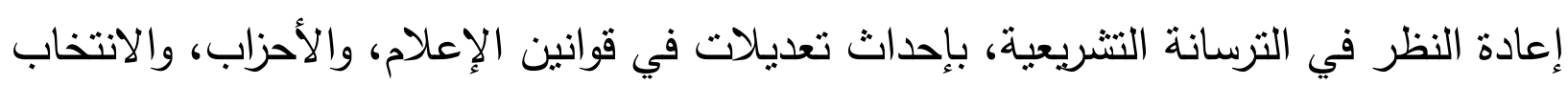
والجمعيات وتقويمها.

-إصلاح القانون العضوي المتعلق بالاتخاب: يعد قانون الانتخاب الإطار القانوني الذي ينظم ويؤطر الفعل الانتخابي،ونظام الحكم في الجزائر تعاقبت عليه عدة أنماط من النظم الانتخابية(نظام الأغلبية،

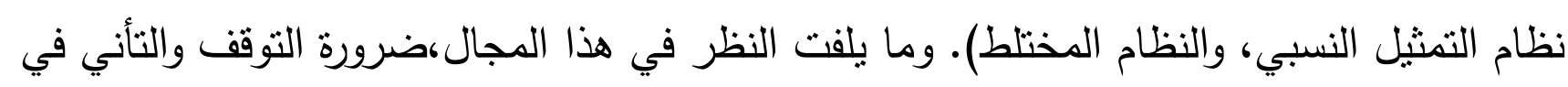
دراسة ما لكل نظام انتخابي وما عليه، من أجل الاختيار الرشيد والعقلاني لأفضل الأنماط وأحسنها في في في إدارة العمليتين السياسية والانتخابية. -إصلاح قانون الأحزاب السياسية: بموجب دستور 1989تم وضع الأسس الأولى لنظام سياسي تعددي، حيث أفرزت الساحة السياسية مواليد سياسية(أحزاب وجمعيات ذات طابع سياسي) وكان عدها معتبرا،

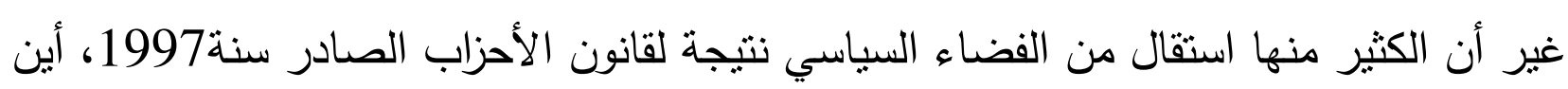
تدخلت المؤسسة القضائية وحلت الكثير منها بحجة عدم قدرتها على التكيف والتأقلم مع الأحكام الجديدة للقانون. وبعد سنة1997 نقدمت العديد من الهيئات والثخصيات السياسية من أجل اعتماد أحزاب جديدة، إلا أنها قوبلت بالرفض من طرف الهيئة المخولة دستوريا بذلك(وزارة الداخلية).أما الفكرة التي

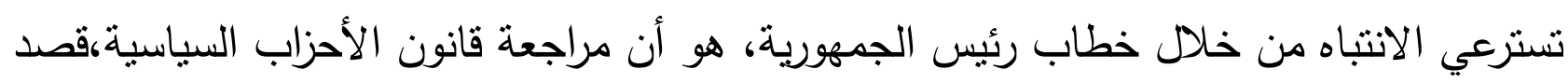
السماح للمعارضة بالنشاط السياسي باعتباره حق مكفول دسنوريا والقانون لا يمنع من تأسيس أحزاب لتلاك الأغراض. -دعم فضاء السمعي البصري: تصمن الخطاب تثمين حرية الصحافة والإعلام، وكذا توسيع الفضاء الإعلامي وإعادة النظر في صياغة السياسة الإعلامية، لتتلاعم أكثر مع بيئة النظام التعددي الديمقراطي، فضلا عن وضع مدونة لأخلاقيات الصحافة والإعلام. -توسيع التمثيل السياسي للمرأة: نم التأكيد على وضع آليات تكفل ضمان تمثيل المرأة في المجالس المنتخبة، والمشاركة بصفة فعالة في العملية السياسية. -توسيع فضاء نشاط الحركة الجمعوية: نظرا لما تتمتع به الحركة الجمعوية من أهمية قصوى في صنع القرار وترشيد سياسة الدولة، فقد أكد الخطاب على إعادة النظر في المنظومة البنائية-الوظيفية للحركة 
الجمعوية، وذلك بفتح المجال أمامها للمشاركة في تفعيل أجهزة الدولة ومحاربة الفساد والرشوة.هذا فضلا

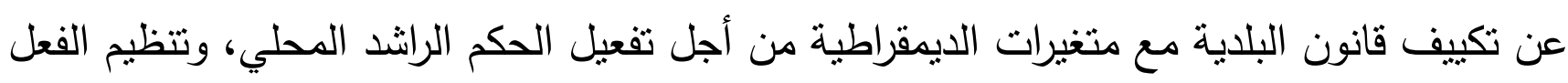
التشاركي للمواطن في صنع القرار والتتمية المحلية.

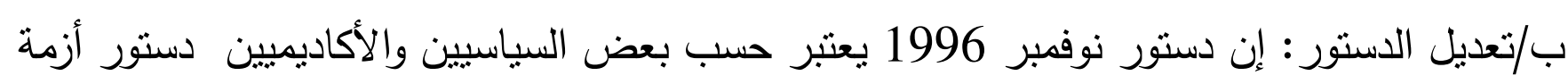
، لذلك كانت من البداية الإرادة السياسية تتوه إلى ضرورة تعديله جوهرانيا، وفعلا كان ذلك سنة 2008،

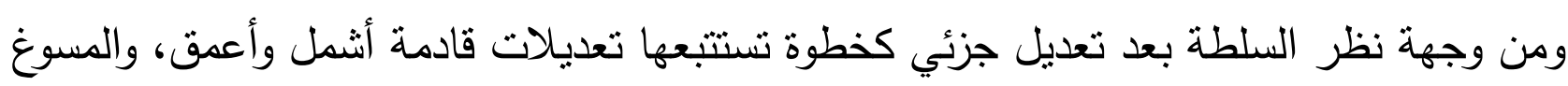
المقدم في هذا الثأن هو أن دستور الأزمة لم يشرك فيه الثنب وفئ وفواعل المجتمع الددني بطريقة مباشرة أو غبر مباشرة.

وتم التركيز من طرف رئيس الجمهورية، على مراجعة دستورية عميقة، دون أن تمس بثوابت الأمة.

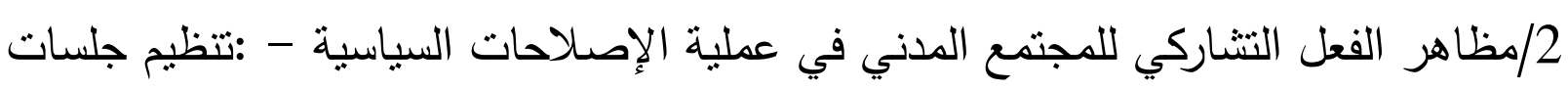

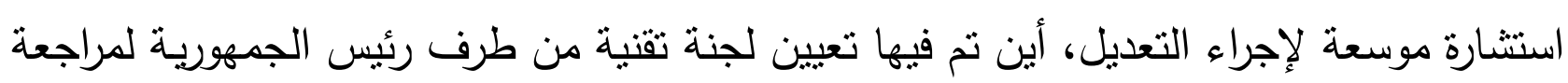
الدستور، وجملة الإصلاحات التثريعية المنتظرة.

وتتثكل لجنة المشاورات من عبد القادر بن صالح رئيس مجلس الأمة والجنرال محمد نواتي

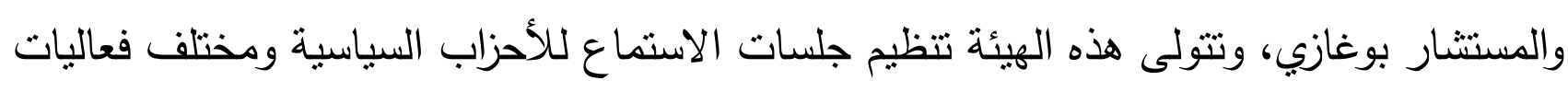

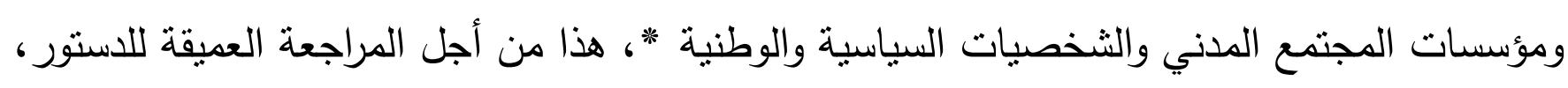

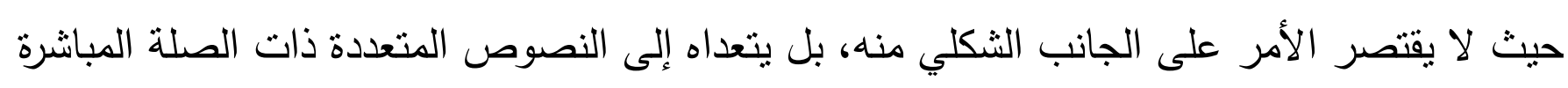

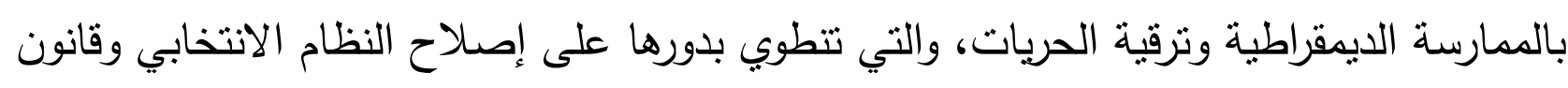

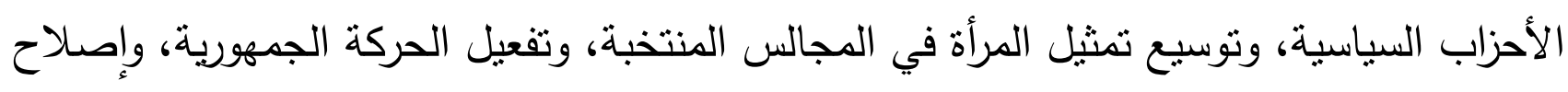

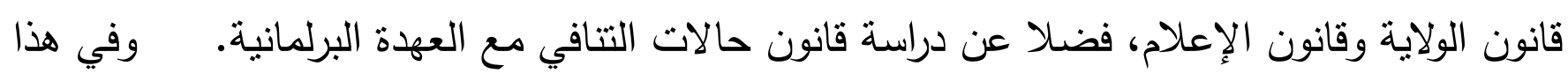

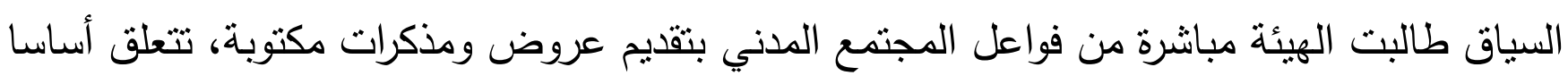

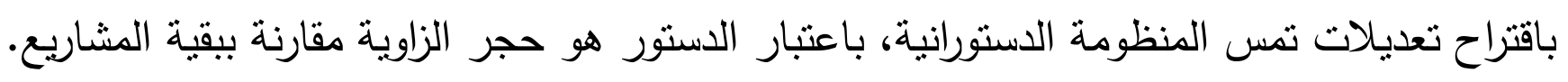

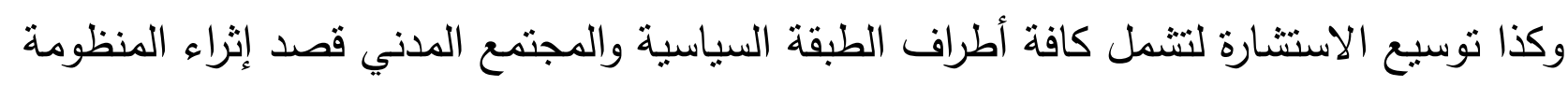
التثريعية المكرسة للإصلاحات.

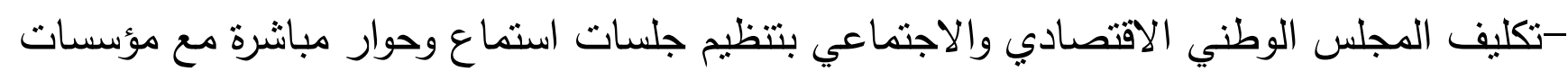

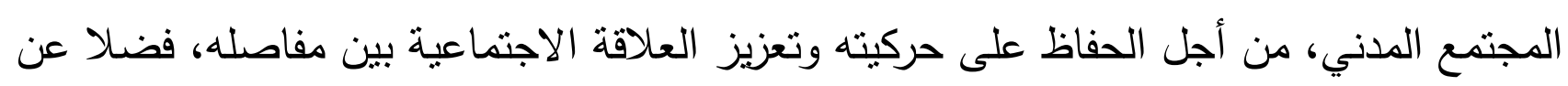

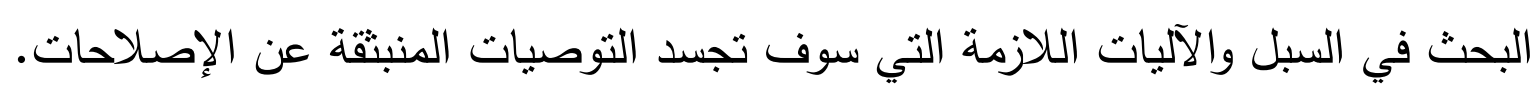


وقد تم خلال الجلسات العلنية المنعقدة بين المجلس الوطني الاقتصادي والاجتماعي ومنظمات المجتمع المدني، التطرق إلى مسألة الإصلاحات عبر خمس ورشات مركزية تتمثل فيما يلي: الورشة الأولى: من أجل نظام جديد للنمو، وجهات نظر للشركاء الاجتماعيين وتتاولت: •الدروس المستخلصة من تجارب 45 سنة من التتمية. •الرهانات والتحديات الراهنة. •اقتراح نموذج جديد للتنمية.

الورشة الثانية: أنظمة الحماية الاجتماعية والتضامن الوطني، شروط ضمانها وديمومتها ـ الورشة الثالثة: من أجل حكم متجدد، حوار اجتماعي، ديمقراطية تشاركية، وتتاولت:

•الحكم وفق المعايير التي وضعتها المنظمات الدولية.

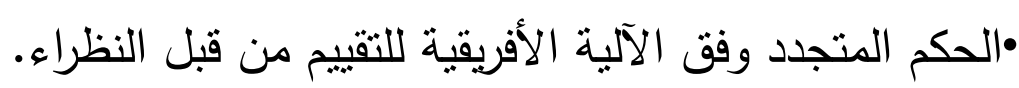
•إصلاح الحوار الاجتماعي للمؤسسات. •تفعيل الديمقراطية النتاركية. الورشة الرابعة: من أجل تكفل حقيقي بإنثكالية الثباب، أثنكال التعبير والتتظيم، قنوات الحوار وتحديث السياسات العمومية.

الورشة الخامسة: تعزيز وتتظيم دور المجتمع المدني: وتم فيها التأكيد على الأهمية البالغة للمجتمع

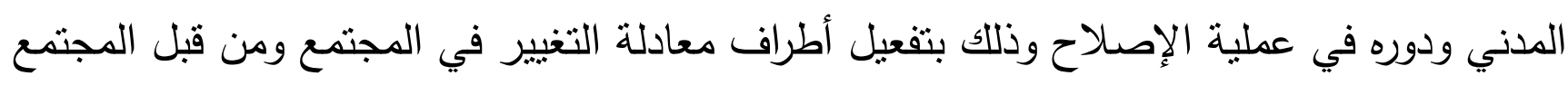
ولأجل المجتمع، من خلال صياغة علاقة ترافقية توافقية بين قاعدة الهرم الاجتماعي والدولة، في إطار مقاربة الديمقراطية التشاركية ، والتي تهدف من خلالها إلى التكيف ومسايرة المتغيرات الإقليمية والدولية لنمط الحكامة الجديد المتضمن المعايير الموضوعة من طرف المنظمات الدولية. 


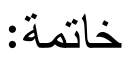

وصفوة القول، من خلال ما تم التطرق إليه عبر صفحات هذه الورقة البحثية الموجزة المتضدنة لتحليل دور المجتمع المدني في التعديل الدستوري، فإنه وعلى الرغم من أثره الواضح نظريا وواقعيا في المساهمة في بناء الدولة تصاعديا لاسيما على مستوى الأنظمة الديمقراطية، غير أنه ييقى مقيد

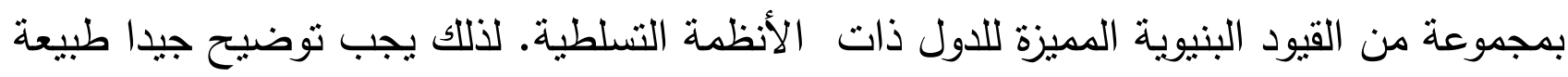
العلاقة جيدا التي تحكم طريقة تفاعل الدولة بالمجتمع المدني، من أجل التأسيس لنقافة دستورية ممأسسة على التكامل والتتسيق وليس الخضوع والابتلاع. 
References:

-1Nasr Mohamed Aref, Comparative Politics Epistemology, 1st edition. (Beirut: University Institute for Studies, Publishing and Distribution, 2002), p. 325.

-2El Ayachi component, reality and understandable civil society - Algeria as an example - for more information see the website:

www.transparency.org.kw.au-ti.org/.../608.pdf

-3Ahmad Shukr Al-Subaihi, The Future of Civil Society in the Arab World, I. 1 (Beirut: Center for Arab Unity Studies, 2000), p. 20.

-4Rabih Wahba, Political Thinking, Political Theory, and Civil Society, I. i. (Cairo: The Supreme Council of Culture, 2003), p. 19.

-5Ahmed Shukr Al-Subaihi, Previous Reference, P.22.

-6Ibid., P. 23.

-7Abdul-Ghaffar Shukr and Muhammad Moro, The Civil Society and its Role in Building Democracy, I.

1 (Damascus: Dar Al-Fikr, 2003). P. 41.

-8Muhammad Abed Al-Jabri, The Problem of Democracy and Society in the Arab World. The Arab Future 167, January 1993 (Beirut: Center for Arab Unity Studies). P. 05.

-9Saad Eddin Ibrahim, Civil Society and Democratic Transition in Egypt (Cairo: Qabaa Printing, Publishing and Distribution House, 2000), p. 13.

-10Ahmed Shukr Al-Subaihi, Previous Reference, pp. 32-33.

-11Zuhair Abdul-Karim Al-Kayed, Al-Hakmaniya Issues and Applications. (Cairo: The Arab Organization for Administrative Development, 2003), p. 48.

-12Mohamed Qadousi, the state and the pure neo-liberal in Algeria after the 1989 constitution continuity, development or interruptions. Journal of Humanities 36. 2008. http://www.ulum.nl

-13Azroual Youssef, Good Governance between Theoretical Foundations and Application Mechanisms A Study in the Reality of the Algerian Experience - (Master's Note in Political Science and International Relations, University of Batna 2009), pp. 96-97.

-14The Official Journal of the People's Democratic Republic of Algeria, November 1996 Constitution, No. 76. 02/08/1996.

-15Al-Youm Newspaper, June 14, 2008, Issue 2478.

-16Abdel Razzaq Mokri, Democratic Transition in Algeria - A Field View.

http://hmsalgeria.net/download/tahawol-dimokrat-algeria.pdf

-17Thamer Kamel Muhammad Al-Khazraji, Modern Political Systems and Public Policies. I 1. (Jordan: Majdalawi Publishing and Distribution House, 2004), p. 292.

-18ibid., P. 293.

*In this regard, more than 241 national, party and group personalities were consulted in sessions that lasted for approximately 45 days (Al-Fajr newspaper, April 15, 2012.(

-19The President's speech to the nation, 15 April 2011.

www.el-mouradia.dz/arabe/president/recherche/recherche.html.

-20Dialogue and meeting sessions organized by the National Economic and Social Council with civil society organizations at the following website: http://www.cnes-forum-civil.dz/Atelier01-Ar.doc http://www.cnes-forum-civil.dz/Atelier02-Ar.doc 21http://www.cnes-forum-civil.dz/Atelier03-Ar.doc 22http://www.cnes-forum-civil.dz/Atelier04-Ar.doc23http://www.cnes-forum-civil.dz/Atelier05-Ar.doc24- 